This article is a post print version.

\title{
How Social and Mass Media Relate to Youth's Self-Sexualization: Taking a Cross- National Perspective on Rewarded Appearance Ideals
}

\author{
Jolien Trekels ${ }^{1}$, Kathrin Karsay ${ }^{2}$, Steven Eggermont ${ }^{1}$, and Laura Vandenbosch ${ }^{1,3}$ \\ ${ }^{1}$ School for Mass Communication Research, Faculty of Social Sciences, KU Leuven, Leuven \\ Belgium \\ ${ }^{2}$ Department of Communication, University of Vienna, Vienna, Austria \\ ${ }^{3}$ Research Foundation Flanders (FWO-Vlaanderen)
}

Please cite as follows:

Trekels, J., Karsay, K., Eggermont, S., \& Vandenbosch, L. (2018). How social and mass media relate to youth's self-sexualization: Taking a cross-national perspective on rewarded appearance ideals. Journal of Youth and Adolescence. Accepted for Publication.

Author note

Email addresses: Jolien.Trekels@kuleuven.be; Kathrin.Karsay@ univie.ac.at; Steven.Eggermont@kuleuven.be; Laura.Vandenbosch@kuleuven.be

Correspondence concerning this article should be addressed to Jolien Trekels, School for Mass Communication Research, KU Leuven, Parkstraat 45, B-3000 Leuven, Belgium, Jolien.Trekels@kuleuven.be, Tel: +32 16323414 -Fax: +32 16320497. 


\begin{abstract}
Although media exposure has been related to cognitive preoccupation with appearance, research rarely investigated adolescents' behavioral self-sexualization. To address this gap, the present study among 12 - to 16 -year-olds $(N=1,527 ; 50.2 \%$ girls $)$ in Austria, Belgium, Spain, and South-Korea (1) investigates whether different types of media use relate to selfsexualization, (2) explores the explanatory value of rewarded appearance ideals, and (3) considers culture and gender as moderating factors. Despite cultural variation, a general trend of increasing self-sexualization with social media use and magazine reading appeared across the countries. Moreover, women's magazine reading and rewards were related to selfsexualization among all the girls across the countries, which suggests that girls may be more vulnerable to the examined effects. Overall, this study provides a better understanding of the unique contribution of specific media genres to youth's self-sexualization and points at the importance of social media use in girls' and boys' engagement in sexualizing appearance behaviors across four countries.
\end{abstract}

Keywords: Traditional media, Social media, Internalization, Rewards, Selfsexualization, Cross-cultural 


\section{Introduction}

Early adolescence is marked as a time of identity development characterized by the importance of expressing one's gendered self (Erikson, 1968; Kroger, 2007). As such, early adolescents are more eager to endorse gender-stereotypical attitudes and engage in gendered behaviors (Hill \& Lynch, 1983; Galambos, Berenbaum, \& McHale, 2009). Mass media have been considered important socializing agents teaching youth about what is considered appropriate for men and women (e.g., Strasburger \& Wilson, 2002). However, concerns have been expressed about contemporary society's focus on and overvaluation of attractiveness and sexiness, i.e., sexualization (APA, 2007). Specifically, content analyses of popular mass media, including prime-time television (e.g., Ganahl, Prinsen, \& Netzley, 2003), music video clips (e.g., Aubrey \& Frisby, 2011), and magazines (e.g., Hatton \& Trautner, 2011) have consistently supported the prevalence of sexualizing and gender-stereotypical messages. Exposure to these kinds of messages encourages the adoption of a narrow view on what women and men can do, especially among youth (Ward \& Harrison, 2005).

Specifically, one potential derivative from media exposure that has received increased scholarly attention is youth's self-sexualization (Ward, 2016). In the present study, we further explored this relation, with two goals in mind. First, we examined whether adolescents become active participants in this sexualizing culture by engaging in self-sexualizing appearance behaviors (e.g., applying make-up for girls or drinking muscle-enhancing beverages for boys). Second, in order to understand whether and how mass media and social media play into youth's self-sexualization, we (1) include adolescents' exposure to multiple media types, (2) pay specific attention to the reward-context in which sexualizing messages occur, and (3) develop a cross-cultural perspective on the role of media in youth's sexualization by comparing Austrian, Belgian, Spanish, and South-Korean adolescents. 


\section{Youth's Exposure to Sexualizing Media Messages}

With an average of two hours of screen time per day, television remains one of the most popular leisure activities during adolescence (e.g., Devís-Devís, Peiró-Velert, BeltránCarrillo, \& Tomás, 2009; Vereecken, Todd, Roberts, Mulvihill, \& Maes, 2006). In addition, social media have become deeply rooted into the daily life of adolescents with $92 \%$ of teens indicating to go online daily, with $24 \%$ of them going online almost constantly (Pew Research Center, 2015). Ample research has linked media exposure and social media use to body image disturbances and gender-stereotypical attitudes (for reviews see, Barlett, Vowels, \& Saucier, 2008; Holland \& Tiggemann, 2016; Levine \& Murnen, 2009). Notably, in 2007, APA invoked a task force warning for the deleterious impact of exposure to sexualizing messages (through media, peers, and family) on adolescent development. Sexualization is defined as occurring when (1) “a person's value comes only from his or her sexual appeal or behavior, to the exclusion of other characteristics; (2) a person is held to a standard that equates physical attractiveness (narrowly defined) with being sexy; (3) a person is sexually

objectified — that is, made into a thing for others' sexual use; (4) sexuality is inappropriately imposed upon a person" (APA, 2007, p. 1). Such messages are commonplace in adolescents' media diet, both in traditional media and social media.

Specifically, sexualizing messages have been shown to be prevalent across various genres of traditional media, including primetime television, music video clips, movies, and magazines. For instance, Kim et al. (2007) coded 25 primetime television programs rated most popular among adolescents. They concluded that all 25 programs contained some element of the heterosexual script (i.e., men have uncontrollable sexual needs that are met by women whose sexuality is solely a means to attract men). In particular, the message that men are sex-driven occurred most frequently (7.01 times per hour), followed by the message that women are valued merely for their appearance (2.08 times per hour). A strong focus on 
women's sexual body parts and adherence to narrowly defined appearance ideals is also abundantly present in music videos. Specifically, content analyses by Aubrey and Frisby (2011) and Frisby and Aubrey (2012) have documented sexualizing messages in music clips and showed that looking sexually alluring was a more important issue for female than male artists. Notably, women have also been depicted as strategically using sexiness as a way to get men's attention in movies that are popular among youth (e.g., Martin \& Kayzak, 2009). Such bias can be problematic as romantic movies are considered important for adolescents to learn about romantic relationships, as they start developing a romantic identity and lack dating experience (Connolly \& McIsaac, 2011).

Magazines have also been argued to sexualize the female and male body by displaying full body shots (Ricciardelli, Clow, \& White, 2010; Sypeck, Gray, \& Ahrens, 2004). Importantly, magazines have also been criticized for sexualizing youth. For instance, Graff, Murnen, and Krause (2013) showed that the number of sexualizing characteristics in images of girls in two teen girls' magazines (Seventeen and Girls' Life) increased over time. Specifically, girls were more often depicted in sexualizing clothing (e.g., low-cut tops and tight clothes), whereas the number of childlike characteristics decreased. A recent evolution has been the adultification of girls. Specifically, Speno and Aubrey (2017) found that girls were adultified (i.e., "dressed up" to look like women) in $84.4 \%$ of images of girls in advertisements and editorials in women's, men's, and teen magazines. Specifically, girls were adultified through provocative dress and sexy facial expressions.

Next to traditional media, researchers have pointed at the importance of including social media in body image research (Perloff, 2014). Recent reports indicate that approximately nine in ten adolescents report being member of at least one social networking site (e.g., Frison \& Eggermont, 2015; Madden, 2013; Tsitsika et al., 2014). However, social media, such as Facebook and Instagram, have been argued to almost exclusively focus on 
appearances as they primarily display visual images (Perloff, 2014). Additionally, sexualization on social media has been increasing (e.g., Kapidzic \& Herring, 2014).

\section{Sexualizing Appearance Behaviors Among Youth}

Prior research has related exposure to sexualizing media messages to adolescents' cognitive preoccupation with outward appearance. Specifically, media exposure has been related to girls' and boys' self-objectification (i.e., valuing body appearance over body competence; Vandenbosch \& Eggermont, 2012), dysfunctional appearance beliefs (i.e., equating self-worth with appearance; Trekels \& Eggermont, 2016), and anxiety to be judged and rejected based on their appearance (e.g., Trekels \& Eggermont, 2017a). Studies have further shown that young girls may accept a narrow and sexualizing view on girls and women as part of their own identity (e.g., McKenney \& Bigler, 2014a). Boys, too, have been shown to internalize and endorse a sexualized view of the self (e.g., Vandenbosch \& Eggermont, 2015). Despite the growing knowledge on the role of media in youth's sexualized self-image, little is known about media's role in behaviors related to sexualization (Ward, Seabrook, Manago, \& Reed, 2015). Among adults, self-sexualization has been defined as "intentionally engaging in activities to appear more sexually appealing" such as wearing cologne, wearing tight clothes, and wearing short skirts/shorts (Smolak, Murnen, \& Myers, 2014, p. 1).

Some prior studies examined young girls' engagement in sexualizing appearance behaviors. For instance, McKenney and Bigler (2014b) found that girls who considered a sexual attractive appearance as an important part of women's value to men, spent more time on appearance-focused behaviors (i.e., applying make-up) than on rehearsing the script in a laboratory task. Recently, Trekels and Eggermont (2017b) also showed that girls were more likely to report engaging in various appearance-management behaviors (e.g., applying makeup and styling their hair) when they frequently watched teen media. Among men, Tod and Edwards (2013) demonstrated that drive for muscularity related to physique-enhancing 
behaviors and that appearance schemas, body image disturbance, and situational body image dysphoria positively moderated this association by eliciting body image cognitive processing.

\section{Explanatory Mechanism}

Researchers have often referred to the internalization of appearance ideals as the mechanism underlying the association between mass media exposure and youth's body image concerns and appearance-altering behaviors (e.g., Stice, 2002). Specifically, it is assumed that individuals make inferences about the benefits for fitting the ideal from being exposed to idealized imagery in mass media and social media. Content analyses have shown that media figures complying with the ideal are depicted as kind (e.g., Herbozo, Tantleff-Dunn, GokeeLarose, \& Thompson, 2004), happy (e.g., Malkin, Wornian, \& Chrisler, 1999), and popular (e.g., Northup \& Liebler, 2010). These expectations of possible "rewards" are theorized to further perpetuate the ideals and affect the extent to which an individual buys into (i.e., internalizes) socially defined appearance ideals and engages in behaviors to approximate these standards (Thompson \& Stice, 2001). This reasoning is in line with Engeln-Maddox's (2006) suggestion that women internalize appearance ideals because they believe it will bring about various rewards. Recently, Trekels and Eggermont (2016) confirmed these assumptions and showed that early adolescents were more likely to internalize appearance ideals if they believed that compliance with those ideals is rewarded.

However, next to depicting a narrow definition of what constitutes an ideal female and male appearance within a rewarding context, mass media are critiqued for presenting such rewarded appearance ideals within gender-stereotypical storylines (Gunter, 2014). As such, the intertwining of sexualizing and gender-stereotypical messages has led researchers to argue that sexualization has become an aspect of youth's socialization into (narrow) cultural gender norms (Zurbriggen \& Roberts, 2013). Specifically, according to the social cognitive theory of gender development and differentiation (Bussey \& Bandura, 1999), adolescents 
come to understand the expected roles of men and women by observing how attractive models engage in and are rewarded for gender-appropriate behavior. Because being liked and accepted is an important issue during adolescence (Blakemore \& Mills, 2014), adolescents might consider mass media as a source to learn about what is expected to be appropriate behavior for men and women. If the rewards are valued by the individual, she/he may be more inclined to imitate the behavior in the hope of reaping the rewards her/himself. It follows that, if girls and boys observe how media figures are rewarded for engaging in appearance-focused behaviors to emulate the ideal female/male appearance (e.g., a woman putting on make-up or a man participating in strength training), they may be more inclined to imitate those behaviors. The theory further argues that adolescents will learn to map the learned gender conception to multiple actions and situations and, for instance, more generally prefer sexualized appearance features (e.g., sexy clothes as opposed to unrevealing clothes).

Following the above, the current study takes a rewards perspective and argues that adolescents' internalization of appearance ideals is affected by their perception of rewards associated with fitting the ideal (i.e., internalization of rewarded appearance ideals; IRAI). Furthermore, IRAI is considered to underlie the association between mass media exposure and adolescents' self-sexualizing appearance behaviors.

\section{Taking a Cross-Cultural View on the Examined Relations}

When it comes to processes explaining how exposure to sexualizing content affects individuals' body image cross-culturally, Moradi (2010) argued the importance of taking into account the cultural divergence in interpretations of the masculine and feminine ideals. Although sexual attractiveness has been found to be an aspect of perceived female attractiveness across Asian, Hispanic, and White participants (e.g., Cunningham, Roberts, Barbee, Druen, \& Wu, 1995), it is argued that an ideal appearance and how it is presented is socially constructed. In other words, what constitutes an ideal appearance might be 
influenced by culture.

Content analyses on beauty ideals across cultures led to mixed findings, though. Specifically, some studies have reported cultural differences in media portrayals of a sexualized appearance. For instance, Yan and Bissell (2014) showed that models in US magazines were more likely to be sexualized and glamourized, whereas models in Asian magazines were less likely to be sexualized. Additionally, Morris and Nichols (2013) found that US models were less sexual than models in French magazines. Such findings indicate that a sexualized appearance may be more prevalent in Western-European countries as opposed to Asian countries. However, at the same time, there are indications that individuals in different cultures are exposed to similar content due to a global culture and a global consumer market (Tan et al., 2013). For instance, American programs such as America's Next Top Model are aired in the US as well as over one hundred other countries, evidencing the global television industry (Bielby \& Harrington, 2008). Still, individuals in different cultures may differently interpret and perceive social norms (Kim \& Aubrey, 2015).

Studies examining how exposure to various types of media may, differently or similarly, affect individuals' endorsement of sexualized appearance ideals across countries are, however, scarce. In fact, in her recent review of the existing literature on media and sexualization, Ward (2016) concluded that most of the studies were conducted among White US college students. The current study aims to address this lacuna in the literature by conducting a cross-cultural examination of the hypothesized relations in Austria, Belgium, Spain, and South-Korea. Theoretically, we can assume that individuals in different cultures may relate differently to normative information (Hofstede, 1983, 2001). Specifically, Hofstede $(1983,2001)$ characterized cultures according to their levels of individualism/collectivism. Individuals living in individualistic countries are thought to primarily identify with the self (i.e., based on one's own needs, preferences, and 
achievements), rendering norms less likely to affect perceptions and behaviors. Collectivistic cultures, on the other hand, are characterized by strong in-group norms along which individuals develop their identity and monitor their behavior. Belgians are considered to be display a strong individualistic orientation. Although Spain and Austria are found to be less strongly focused on the individual, they are still considered to be part of the Western society (Hofstede, 2001). South-Korea, on the other hand, is considered be to a collectivistic society (Hofstede, 2001). Such cultural dimensions may, then, reveal variations in the extent to which individuals endorse and move towards achieving the prevailing ideals.

Although empirical studies conducting such cross-cultural comparisons are scarce, there are indications that taking on a cross-cultural perspective is valuable. For instance, Gattino, de Piccoli, Fedi, Boza, \& Rollero (2017) found that self-objectification was mostly influenced by sociocultural factors (e.g., internalization of media ideals) among Italian adults, whereas Romanian adults experienced self-objectification through individual characteristics (such as self-esteem). Kim and Aubrey (2015) also observed that the association between thin-ideal media use and body image disturbances was mediated by normative beliefs about thinness among both US and Korean women, but the effect was stronger among Korean participants.

\section{Considering the Role of Gender in the Examined Relations}

Apart from cross-cultural differences, the literature has pointed at the potential important role of gender. Yan and Bissell (2014) showed that the cover of four top fashion and beauty magazines that are published worldwide (i.e., Vogue, Elle, Glamour, and Cosmopolitan) features a model that adheres to narrowly defined standards of ideal beauty. Additionally, cover models were highly feminine, glamorized, and sexualized across all countries and cover stories are aimed to convince readers that these ideals are desirable and attainable. Following such findings, it has been argued that girls may be more embedded in 
an appearance culture and experience more pressure to comply with appearance ideals than boys (e.g., Jones, Vigfusdottir, \& Lee, 2004). Girls have, in fact, been shown to more frequently engage in behaviors to alter their appearance, such as dieting, than boys (e.g., McCabe \& Ricciardelli, 2006). Nevertheless, boys also report endorsing narrowly defined ideals as personal standards (e.g., Vandenbosch \& Eggermont, 2015) and engage in muscleenhancing behaviors to emulate an increasingly muscular male ideal (e.g., McCabe \& Ricciardelli, 2005). With regard to cross-cultural differences, one study (Paek et al., 2011) documented the underrepresentation of women in ads and showed that this trend occurs across various countries (i.e., Germany, US, China, Canada, Brazil, and Thailand). Conversely, more women were portrayed as prominent characters than men in ads in SouthKorea. Still, the way men and women are portrayed in South-Korea, as in the other countries, adheres to traditional gender roles with men being depicted in professional occupations, while women were more likely to be featured as homemakers (Paek, Nelson, \& Vilela, 2011).

\section{The Present Study}

The primary goal of the present cross-cultural study among 12- to 16-year-olds was to examine whether different types of media use relate to adolescents' engagement in selfsexualizing appearance behaviors (Hypothesis 1). Notably, adolescents' media diet comprises various genres and it could be expected that each genre uniquely contributes to sexualization (Vandenbosch \& Eggermont, 2012). Specifically, men and women are sexualized differently on television than they are in magazines. On television, men and women are involved in sexualizing storylines (e.g., Kim et al., 2007) whereas magazines focus on teaching readers how to emulate idealized and sexualized looks (e.g., Yan \& Bissell, 2014). Moreover, social media provide another unique perspective on sexualization by displaying sexualized images of peers, whom are unique and important comparison targets for youth (Manago, Ward, Lemm, Reed, \& Seabrook, 2015). Therefore, Research Question 1 explores whether there are 
any differences between media genres with regard to their association with self-sexualizing appearance behaviors.

An additional goal of the current study is to further disentangle the association between media exposure and self-sexualization by (1) exploring an underlying mechanism and (2) considering two moderating factors (i.e., culture and gender). First, although some studies did not find support for adolescent gender intensification (e.g., Priess, Lindberg, \& Hyde, 2009), there are indications in the literature that adolescents are especially vulnerable to the sociocultural reinforcement of gendered appearance ideals because they are likely to reach out to mass media to learn about the expected gender roles (Arnett, 1995). Therefore, the current study builds on prior body image literature (Thompson \& Stice, 2001) and social cognitive theory (Bandura, 2001) to take a rewards perspective on sexualizing media effects. Specifically, it is argued that the rewards associated with fitting the ideal affects adolescents' internalization of appearance ideals (i.e., IRAI) which may underlie the association between media exposure and adolescents' self-sexualization (Hypothesis 2).

Second, drawing on earlier research (e.g., Gattino et al., 2017) which has supported the value of taking a cross-cultural perspective to understand sexualizing media effects, we will compare the examined relations across participants in Austria, Belgium, Spain, and South-Korea (Research Question 2). Finally, based on prior findings (e.g., Mccabe \& Ricciardelli, 2006) which has shown that girls may be especially vulnerable to the negative impact of media exposure on their body image and appearance-related behaviors, the moderating role of gender - within countries - will be examined (Research Question 3).

\section{Method}

\section{Participants}

Between February and May 2017, a large-scale cross-sectional study (called the Intercultural Study Project, ISP) was conducted in Austria, Belgium, Spain, and South- 
Korea. ${ }^{1}$ The ethical committees of each host university approved the study. The study targeted early and middle adolescents (12- to 16-year-olds) in each country. For this purpose, we contacted schools in each country to participate in the study until approximately 300 adolescents would participate in each country. In all, seven schools in Austria, eleven schools in Belgium, four schools in South Korea, and five schools in Spain agreed to participate. After active consent was obtained, adolescents filled out paper-and-pencil surveys in class during class hours. Researchers were present at all times to ensure optimal circumstances by making sure all participants could fill in the questionnaire privately and in silence. Moreover, participants were guaranteed anonymity and confidentiality during participation in the study. A total 1,983 adolescents (993 or $50.3 \%$ boys) filled out the questionnaire $\left(M_{\text {age }}=14.41\right.$ years; age range $=12-19 ; S D=1.08)$. Frequency distribution across countries was as follows: Austria: $N=368,18.6 \%$; Belgium: $N=675,34.0 \%$; Spain: $N=564,28.4 \%$; South-Korea: $N$ $=376,19.0 \%$. However, some participants had missing values on the key variables and were omitted from the analyses. The analytical sample contained 1,527 adolescents.

\section{Measures}

The questionnaire was initially constructed in English. Next, researchers translated the questionnaire into their own native language (i.e., German, Dutch, Spanish, and Korean) and ensured the translation was correct by asking other experienced researchers to review the questionnaire and, if necessary, correct the translated questions.

Socio-demographic variables. We included gender ( 1 = "boy", 2 = "girl”), country (1 = "Austria", 2 = "Belgium", 3 = "Spain", 4 = "South-Korea"), and age as sociodemographic variables. Age was included as a control variable because adolescence is

\footnotetext{
${ }^{1}$ The current article uses data that is part of a larger intercultural study project that examines links between media usage and well-being among adolescents in four different countries. More information about the study project can be obtained by sending an email to the first author.
} 
characterized by the onset (early adolescence) and completion (middle adolescence) of pubertal changes which bring about a heightened awareness of one's overall physical appearance (Williams \& Currie, 2000). Notably, adolescent self-concept and perceptions of appearance are inextricably linked (e.g., Zsakai, Karkus, Utczus, \& Bodzar, 2017).

Importantly, the timing of pubertal development typically differs between girls and boys and cross-nationally (Parent et al., 2003), and may therefore be important to include as a control variable.

In addition, research suggests that BMI affects the development of a sexualized selfconcept and appearance management (Harrison and Fredrickson, 2003; Neumark-Sztainer \& Hannen, 2000), and was therefore also included (based on respondents' self-reported height and weight).

Media use. Respondents indicated how much time per day they usually spent on average on social media (i.e., Facebook, Instagram, Snapchat, and WhatsApp), watching music video clips, watching prime time television programs (i.e., television programs aired between $6 \mathrm{pm}$ and $11 \mathrm{pm}$ ), watching romantic movies or dramas, reading women's and fashion magazines, and men's magazines. Response categories were: "never use it to less than 10 min”, “10-30 minutes”, “31-60 minutes”, "1-2 hours”, “3-4 hours”, "5-6 hours”, "more than 6 hours."

The internalization of rewarded appearance ideals. To examine whether adolescents internalize appearance ideals because they believe an ideal appearance is associated with rewards or benefits, we created a new scale inspired by the Internalization subscale of the Sociocultural Attitudes towards Appearance Scale (Thompson, Van Den Berg, Roehrig, Guarda, \& Heinberg, 2004). Specifically, respondents used a 5-point scale ranging from "I totally disagree" $(=1)$ to "I totally agree" $(=5)$ to rate whether they would like their body to look like the people who appear in magazines and on television because "it 
would boost their self-confidence," "they would be more popular with friends," "they would get a girl/boyfriend more easily," "they would be more happy," and "they would feel better about themselves." A principal component analysis yielded one factor (eigenvalue: 3.46; explained variance: $69.17 \%$ ). Internal consistency of the scale was established for the entire sample, and separately for girls and boys $\left(\alpha=.89, \alpha_{\text {girls }}=.88\right.$ and $\left.\alpha_{\text {boys }}=.90\right)$.

Self-sexualizing behaviors. To assess adolescents' engagement in self-sexualizing behaviors, we followed Trekels and Eggermont (2017c) and asked adolescents to indicate how often they performed each of several behaviors on a scale ranging from "Never" $(=1)$ to "Always" (=5). These behaviors were chosen based on prior research by Smolak, Murnen, and Myers (2014), and on literature on the sexualization of youth (e.g., APA, 2007;

Zurbriggen \& Roberts, 2013), and were adapted to fit the age of our respondents. For boys, these behaviors included: wearing tight clothes, wearing low-cut shirts that expose the chest, enjoying walking around in an undressed upper-body, wearing pants so that underwear is visible, exercising to get a good physique, drinking beverages to gain muscle, and styling their hair. A principal component analysis yielded one factor (eigenvalue: 3.12; explained variance: $44.53 \%$ ). Reliability analysis revealed that omitting the item "exercising to get a good physique" would increase reliability $(\alpha=.80)$. As a result, the scale for boys included six behaviors. Items were averaged to get an overall measure of engagement in selfsexualizing appearance behaviors. For girls, we asked how often they style their hair, dye their hair, apply make-up, wear heels, wear tight clothes, wear short skirts/shorts, exercise to get a good physique, and wear perfume. A principal component analysis yielded one factor (eigenvalue: 2.88 ; explained variance: $35.96 \% ; \alpha=.72$ ). Subsequently, items were averaged. These results are comparable to other studies using this scale (Trekels \& Eggermont, 2017c).

\section{Data Analysis Plan}

First, measurement invariance for the IRAI scale was examined. Specifically, the 
IRAI scale used scale measurements, which were used as latent variable indicators in the SEM model (following Trekels and Eggermont (2017c) self-sexualization was entered as a manifest variable rendering a measurement invariance test unnecessary). Measurement invariance for the IRAI scale was tested through a multiple group analysis. CFI values (Cheung \& Rensvold, 2002) were used to determine whether groups were invariant with regard to the measure. If the difference in CFI values is less than .01 between the unconstrained (i.e., factor loadings vary freely) and the constrained model (i.e., factor loadings are constrained to be equal) than the hypothesis of invariance is accepted. The results showed that $\Delta$ CFI was $.011(.980-.969)$. Further examination of the separate factor loadings showed, however, that factor loadings did not differ significantly between individuals from the four countries.

Next, we tested the proposed model through structural equation modeling in AMOS, using the maximum likelihood method. We used the chi-squared-to-degrees-of-freedom ratio $\left(\chi^{2} / \mathrm{df}\right)$, the comparative fit index (CFI), the root mean square error of approximation (RMSEA), and the Tucker-Lewis index (TLI) to address the fit of the models. The model was first tested with the full sample $(N=1,527)$.

To test whether the hypothesized relations differed between (1) participants from the different countries, and (2) girls and boys, we conduced multiple group comparison tests. Specifically, for country and for gender within countries, we compared a model in which all parameters are allowed to vary across groups (i.e., unconstrained model) with a model in which all parameters are fixed to be equal across groups (i.e., constrained model). If this test was significant, subsequent path-by-path analyses were performed to examine which relations differed significantly between the groups. For country, this multiple-group analysis was performed comparing four groups (i.e., Austrian $[N=263]$, Belgian [ $N=485]$, Spanish $[N=438]$, and South-Korean [ $N=341]$ participants). For gender, this multiple-group 
analysis was performed comparing eight groups (i.e., Austrian girls $[N=144]$ and boys $[N=$ 119], Belgian girls $[N=208]$ and boys [ $N=277]$, Spanish girls $[N=250]$ and boys $[N=$ 188], and South-Korea girls $[N=164]$ and boys $[N=177])$.

\section{Results}

\section{Preliminary Analyses}

Zero-order correlations and descriptive statistics for the entire sample are presented in Table 1. Through a MANOVA analysis (using Pillai's Trace), we found that adolescents from the different countries differed on all key variables, $F(24,4554)=43.88, p<.001, \eta p^{2}=$ .19. The results are presented in Figure 1.

\section{Overall Model}

The proposed model was first tested with the full sample $(N=1,527)$ and showed a tolerable fit of the data with $\chi^{2} / \mathrm{df}=10.01, p<.001, \mathrm{CFI}=.94, \mathrm{TLI}=.85, \mathrm{RMSEA}=.08$. Music video clips $(\beta=.13, B=.08, S E=.03,95 \%$ CI $[.02, .14], p=.007)$, women's magazines $(\beta=.08, B=.17, S E=.07,95 \% \mathrm{CI}[.02, .14], p=.008)$, and prime time television $(\beta=.09, B=.09, S E=.03,95 \%$ CI $[.04, .15], p=.002)$ were significantly related to IRAI. IRAI was, however, not related to self-sexualization $(\beta=.03, B=.02, S E=.02,95 \%$ CI [-.02, $.08], p=.242)$. The results further showed that women's magazines $(\beta=.15, B=.19, S E=$ $.03,95 \% \mathrm{CI}[.09, .20], p=.002)$ and social media $(\beta=.28, B=.19, S E=.02,95 \%$ CI $[.23$, $.33], p=.002$ ) were directly related to self-sexualizing behaviors.

\section{Hypothesized Model Across Countries}

Differences among participants from the different countries were explored by running multiple group comparisons (Figure 2). Both models showed a good fit of the data: unconstrained model (i.e., model where the parameters vary between the groups): $\left(\chi^{2}(176)=\right.$ 456.04, $\chi^{2} / \mathrm{df}=2.59, p<.001 ; \mathrm{CFI}=.96 ; \mathrm{TLI}=0.91 ; \mathrm{RMSEA}=.03 ;$ constrained model (i.e., model where the parameters are constrained to be equal): $\chi^{2}(230)=735.62, \chi^{2} / \mathrm{df}=3.20, p<$ 
$.001 ; \mathrm{CFI}=.93 ; \mathrm{TLI}=0.88 ; \mathrm{RMSEA}=.04$. A model comparison test between the unconstrained and constrained model was significant, indicating that the processes differed across countries, $\Delta \chi^{2}=77.35, \Delta d f=12, p=.000$. The significant paths per country are summarized in Figure 2.

Association between media use and internalization. First, differences were revealed in the association between different media genres and internalization. A path-by-path analysis showed that the countries differed significantly with regard to the association between social media and IRAI. More specifically, this association was only significant among Austrian adolescents $(\beta=.24, B=.29, S E=.09,95 \%$ CI $[.09, .38], p=.003)$, but not among Belgian $(\beta$ $=.03, B=.03, S E=.05,95 \% \mathrm{CI}[-.08, .13], p=.612)$, Spanish $(\beta=.06, B=.07, S E=.07$, $95 \% \mathrm{CI}[-.06, .17], p=.32)$, or South-Korean adolescents $(\beta=.01, B=.01, S E=.11,95 \% \mathrm{CI}$ $[-.12, .12], p=.897)$. Moreover, the results showed that the association between prime time television and IRAI was significant among Belgian $(\beta=.13, B=.12, S E=.05,95 \% \mathrm{CI}[.02$, $.21], p=.018)$ and Spanish adolescents $(\beta=.12, B=.11, S E=.06,95 \%$ CI $[.01, .23], p=$ $.027)$, but not among Austrian $(\beta=-.08, B=-.07, S E=.07,95 \% \mathrm{CI}[-.22, .06], p=.25)$ and South-Korean adolescents $(\beta=.12, B=.12, S E=.06,95 \%$ CI $[-.01, .23], p=.077)$.

Association between media use and self-sexualization. Second, some media genres related to self-sexualization in some countries but not in other countries. Music video clips related to self-sexualization, among Belgian adolescents only $(\beta=.13, B=.12, S E=.05$, $95 \% \mathrm{CI}[.02, .22], p=.017)$ but not among Austrian $(\beta=-.06, B=-.06, S E=.07,95 \% \mathrm{CI}[-$ $.19, .08], p=.415)$, Spanish $(\beta=.03, B=.04, S E=.05,95 \% \mathrm{CI}[-.06, .15], p=.502)$, nor South-Korean adolescents $(\beta=.02, B=.03, S E=.06,95 \%$ CI $[-.11, .14], p=.687)$. Women's magazines related significantly to self-sexualization among adolescents in Belgium $(\beta=.08$, $B=.11, S E=.05,95 \% \mathrm{CI}[-.001, .15], p=.054)$, Spain $(\beta=.21, B=.24, S E=.05,95 \% \mathrm{CI}$ $[.11, .31], p=.002)$, and South-Korea $(\beta=.34, B=.22, S E=.06,95 \%$ CI $[.23, .46], p=.001)$, 
but not in Austria $(\beta=.06, B=.06, S E=.06,95 \% \mathrm{CI}[-.06, .16], p=.354)$. Additionally, the association between social media and self-sexualization was significant among adolescents in Austria $(\beta=.29, B=.16, S E=.06,95 \%$ CI $[.15, .39], p=.003)$, Belgium $(\beta=.24, B=.10, S E$ $=.04,95 \%$ CI $[.16, .32], p=.004)$, and South-Korea $(\beta=.21, B=.14, S E=.05,95 \%$ CI $[.11$, $.30], p=.001)$, but not in Spain $(\beta=-.06, B=-.04, S E=.05,95 \%$ CI $[-.16, .04], p=.257)$.

Association between IRAI and self-sexualization. Lastly, IRAI related significantly to self-sexualization among adolescents in Austria $(\beta=.22, B=.10, S E=.03,95 \% \mathrm{CI}[.11$, $.33], p=.001)$, Belgium $(\beta=.42, B=.18, S E=.02,95 \%$ CI $[.35, .49], p=.002)$, and SouthKorea $(\beta=.13, B=.05, S E=.04,95 \%$ CI $[.05, .22], p=.004)$, but not in Spain $(\beta=.06, B=$ $.03, S E=.05,95 \% \mathrm{CI}[-.04, .17], p=.62)$.

\section{Hypothesized Model Across Gender in Countries}

We compared the proposed model across gender while differentiating between countries (Figure 3). In other words, boys and girls from each country were compared to explore whether gender moderated the examined relations. We confirmed that there was measurement invariance; $\triangle \mathrm{CFI}$ value was less than the recommended .01 cutoff point (CFI for unconstrained model $=.963 ;$ CFI for constrained model $=.961)$. Subsequently, we examined whether the hypothesized model was moderated by gender through a multi-group analysis. The model fit indices for both models were: unconstrained model $\left(\chi^{2}(496)=\right.$ 2441.27, $\left.\chi^{2} / \mathrm{df}=4.92, p<.001 ; \mathrm{CFI}=.73 ; \mathrm{TLI}=0.74 ; \mathrm{RMSEA}=.05\right)$ and constrained model $\left(\chi^{2}(503)=2497.96, \chi^{2} / \mathrm{df}=4.96, p<.001 ; \mathrm{CFI}=.92 ; \mathrm{TLI}=0.60 ; \mathrm{RMSEA}=.05\right) . \mathrm{A} \chi^{2}$ model comparison test was significant, indicating that the processes differed across gender, $\Delta \chi^{2}=$ $22.16, \Delta d f=4, p=.000$.

A path-by-path analysis showed that IRAI and self-sexualization were significantly related among the girls in all countries (Austria: $\beta=.32, B=.14, S E=.03,95 \% \mathrm{CI}[.17, .27], p$ $=.002$; Belgium: $\beta=.42, B=.18, S E=.03,95 \% \mathrm{CI}[.28, .52], p=.003$; Spain: $\beta=.19, B=$ 
$.09, S E=.03,95 \% \mathrm{CI}[.10, .28], p=.003$; South-Korea: $\beta=.18, B=.08, S E=.04,95 \% \mathrm{CI}[.03$, .32], $p=.028)$. This association was only significant among Belgian boys $(\beta=.39, B=.17$, $S E=.02,95 \% \mathrm{CI}[.27, .49], p=.004)$. The association between women's magazines and selfsexualization was only significant among Belgian $(\beta=.18, B=.17, S E=.05,95 \% \mathrm{CI}[.07$, $.31], p=.002)$, Spanish $(\beta=.29, B=.25, S E=.06,95 \% \mathrm{CI}[.19, .38], p=.003)$, and SouthKorean girls $(\beta=.26, B=.23, S E=.08,95 \% \mathrm{CI}[.13, .37], p=.003)$. Lastly, social media use was related to self-sexualization among both girls (Austria: $\beta=.33, B=.19, S E=.04$, 95\%CI[.18, .49], $p=.001$; Belgium: $\beta=.15, B=.06, S E=.02,95 \% \mathrm{CI}[.03, .28], p=.010$; Spain: $\beta=.14, B=.09, S E=.04,95 \% \mathrm{CI}[.05, .23], p=.002$; South-Korea: $\beta=.27, B=.17$, $S E=.05,95 \%$ CI $[.09, .44], p=.007$ ) and boys (Austria: $\beta=.19, B=.11, S E=.06$, 95\%CI[.04, .35], $p=.015$; Belgium: $\beta=.29, B=.13, S E=.02,95 \% \mathrm{CI}[.19, .40], p=.003$; Spain: $\beta=-.35, B=-.21, S E=.04,95 \% \mathrm{CI}[-.56,-.16], p<.001$; South-Korea: $\beta=.22, B=$ $.13, S E=.05,95 \% \mathrm{CI}[.09, .32], p=.003)$. None of the media variables related significantly to the IRAI among girls nor boys.

\section{Additional Analyses}

Age differences across countries. To understand whether age moderated the examined relations, we compared early ( $<14$ year olds) to middle $(>15$ year olds $)$ adolescents with regard to the examined relations (across the countries). First, we confirmed that there was measurement invariance; $\Delta \mathrm{CFI}$ value was less than the recommended .01 cutoff point $(\mathrm{CFI}$ for uncontrained model $=.944$; CFI for contrained model $=.993)$. The groups could therefore be compared. Subsequently, we examined whether the hypothesized model was moderated by gender through a multi-group analysis. The results showed that the unconstrained model (i.e., model where the parameters vary between the groups) differed significantly from the constrained model (i.e., model where the parameters are constrained to be equal), indicating that the processes differed across age, $\Delta \chi^{2}=49.74, \Delta d f=23, p=.005$. A 
path-by-path analysis showed that early and middle adolescents only significantly differed with regard to the association between the internalization of rewarded appearance ideals and self-sexualization. Specifically, this association was significant among the early adolescents $(\beta=.11, B=.06, S E=.03,95 \% \mathrm{CI}[.04, .17], p=.004)$ but insignificant among the middle adolescents $(\beta=-.05, B=-.03, S E=.04,95 \%$ CI $[-.13, .03], p=.210)$.

Alternative model. An inverse model that assessed the potential influence of selfsexualization and IRAI on the use of different media was tested. The model showed a poorer fit of the data with $\chi^{2}(63)=1609.77, \chi^{2} / \mathrm{df}=25.55, p<.001 ; \mathrm{CFI}=.79 ; \mathrm{TLI}=0.60 ; \mathrm{RMSEA}$ $=.13$. Nevertheless, the model revealed that adolescents' engagement in self-sexualizing behaviors related to their consumption of music videos $(\beta=.07, B=.11, S E=.04,95 \% \mathrm{CI}$ $[.02, .12], p=.010)$, romantic movies $(\beta=.05, B=.09, \mathrm{SE}=.04,95 \% \mathrm{CI}[.00, .10], p=.042)$, fashion magazines $(\beta=.16, B=.13, S E=.02,95 \% \mathrm{CI}[.11, .22], p=.002)$, and social media $(\beta=.27, B=.39, S E=.03,95 \% \mathrm{CI}[.22, .32], p=.002)$. Additionally, IRAI related significantly to music video exposure $(\beta=.13, B=.12, S E=.02,95 \% \mathrm{CI}[.07, .18], p=.002)$, romantic movies $(\beta=.10, B=.10, S E=.03,95 \% \mathrm{CI}[.05, .155], p=.002)$, fashion magazines $(\beta=.12, B=.06, S E=.01,95 \% \mathrm{CI}[.07, .17], p=.002)$, men's magazines $(\beta=.08, B=.02$, $S E=.01,95 \% \mathrm{CI}[.03, .14], p=.003)$, and prime time television exposure $(\beta=.14, B=.01$, $S E=.03,95 \%$ CI $[.07, .19], p=.004)$. Adolescents' engagement in self-sexualizing behaviors did not relate to IRAI $(\beta=.05, B=.08, S E=.05,95 \%$ CI $[-.00, .11], p=.064)$.

\section{Discussion}

Recent studies have shown that mass media exposure relates to girls' and boys' cognitive preoccupation with outward appearance (e.g., Trekels \& Eggermont, 2016; Vandenbosch \& Eggermont, 2012). However, many of these studies have ignored adolescents' active (i.e., behavioral) participation in a sexualizing culture (Ward, 2016) to reap certain benefits. Given the eagerness of adolescents to comply with normative standards 
(Blakemore \& Mills, 2014), and the fact that these normative standards for appearance are presented in a sexualizing and rewarding context (APA, 2007), questions arise as to whether and how different media genres (i.e., traditional media and social media) relate to adolescents' appearance behaviors. To address these questions, we tested the correlational relation between media use and adolescents' self-sexualizing behaviors, through the internalization of rewarded appearance ideals. In addition, studies exploring the applicability of this model across cultures are lacking. To fill this gap, we examined the hypothesized relations among adolescents in Austria, Belgium, Spain, and South-Korea. We believe our study has several important take-home messages.

\section{Direct Associations Between Media Genres, IRAI, and Self-Sexualization}

Our first research question was whether different media genres, including both traditional and social media, are directly related to adolescents' engagement in selfsexualizing appearance behaviors. The results confirmed our expectations and showed that women's magazines and social media were positively related to self-sexualizing behaviors. As such, we found support for the first hypothesis - media exposure relates to adolescents' self-sexualization - and showed some unique and significant contributions of specific media genres (i.e., women's magazines and social media) to youth's self-sexualization (Research Question 1). By including social media in addition to screen and print media, we believe we answered and expanded upon Ward et al.'s (2015) call for more research exploring the relative contribution of specific media genres to self-sexualization. Although more research is necessary to fully understand why certain media genres relate to self-sexualization whereas other do not (e.g., content), we believe our findings are in line with prior studies. For instance, research on the role of social media has reported that the use of SNSs is associated with adolescents' body image investment and disordered eating (Holland \& Tiggemann, 2016). Our findings add that social media use relates to adolescents' inclination to 
behaviorally invest in a sexualized appearance. Additionally, a recent meta-analysis on the association between sexualizing media use and self-objectification showed that media type moderated the effect size (Karsay, Knoll, \& Matthes, 2017). More specific, the effect was stronger for online media (and video games) as compared to television, which is in line with our findings.

Moreover, it was found that music video clips, women's magazines, and prime time television were significantly related to IRAI. However, IRAI - in turn - was not related to self-sexualization. As such, the second hypothesis was not confirmed for the overall sample, rendering it especially relevant to explore the conditions (i.e., culture and gender) under which media use and rewarded appearance ideals may be related to self-sexualization.

With regard to the direct associations, it is important to acknowledge that the correlational design of the current study precludes us from making any inferences about temporal and causal order. An additional analysis assessing the inverse relations showed support for the association between adolescents' engagement in sexualizing behaviors, IRAI, and consumption of different media, implying that adolescents might search for media to confirm their preoccupation with appearance. Future studies applying an experimental or longitudinal design are necessary to further fine-tune scholarly knowledge of how adolescents' media exposure and self-sexualization are related.

\section{The Moderating Role of Culture}

With regard to the moderating role of culture (Research Question 2), support for the association between media use, IRAI, and self-sexualizing appearance behaviors among adolescents seemed scattered across the countries. For instance, social media use was only related to Austrian adolescents' belief that complying with appearance ideals is rewarded. Furthermore, exposure to romantic movies was only related to South-Korean adolescents' engagement in self-sexualizing appearance behaviors. Lastly, exposure to music videos was 
positively associated with Belgians' IRAI and engagement in self-sexualizing appearance behaviors.

Nevertheless, because there were several cross-cultural similarities in the hypothesized relations, we believe that perhaps the differences that were found across cultures can merely be explained by the popularity of certain media types in certain countries. The fact that there are cross-cultural similarities is further in line with the idea that people of diverse cultures may be exposed to the same content; Youth may likely endorse a common set of values because of global television and the internet (Gooderham \& Nordhaug, 2002).

Three main conclusions can be drawn from the cultural perspective on the examined relations. A first conclusion relates to the direct association between media genres and selfsexualization. Specifically, it was found that social media use related significantly and directly to self-sexualizing appearance behaviors among Belgian, Austrian, and SouthKorean adolescents, and among boys as well as girls. This finding coincides, first, with prior research on the importance of social media in youth's lives (Subrahmanyam \& Greenfield, 2016). In our study, Instagram was the most popular social media format, followed by WhatsApp and Facebook. On average, adolescents reported to spend somewhere between half an hour to an hour per day on social media, which may seem relatively low when compared to other research. Specifically, reports often conclude that adolescents spend approximately two hours per day on social networking sites (e.g., Frison \& Eggermont, 2015; Tsitsika et al., 2014). Research among Korean respondents has found more mixed results with usage time ranging from 20 minutes (e.g., Park, Song, \& Lee, 2014) to two hours per day (e.g., Lee, Lee, \& Kwon, 2011). Most of these studies, however, focused on the use of social networking sites, whereas our study included Facebook, Snapchat, WhatsApp, and Instagram. Our focus on various social media formats might explain a different average usage time among adolescents. Specifically, although social media such as Snapchat and Instagram 
are becoming increasingly popular, research indicates that they may still be used less frequently than Facebook (e.g., Pew Research Center, 2015; Utz, Muscanell, \& Khalid, 2015). Second, both Instagram and Facebook are characterized by a focus on physical appearance (Perloff, 2014) and the display of increasingly sexualized images (Kapidzic \& Herring, 2014), which has spurred research into the influence of social media use on adolescents' body image. This research has often concluded that idealized images of peers may be more important factors influencing youth's body image than media figures (Manago et al., 2015). In this regard, Cohen and Blaszcynski (2015) showed that Facebook use was more strongly related to body dissatisfaction and eating disorders than traditional media. In their meta-analysis, Karsay et al. (2017) also showed that online media led to higher levels of self-objectification when compared to traditional media (i.e., television and print media). Our study confirms these findings by showing that time spent on social media was directly related to adolescents' self-sexualization, whereas exposure to prime time television, romantic movies, music videos, and men's magazines was not.

Spanish adolescents appeared to be an exception with regard to the association between social media and self-sexualizing appearance behaviors. We believe this might be explained by the way in which engagement in self-sexualizing appearance behaviors was measured. Specifically, the results showed that Spanish adolescents scored the highest on this measure, which is not surprising given that girls and boys were asked how often they, among other things, wore short skirts or shorts. Due to the warm climate in Spain, adolescents may have indicated to wear short skirts/shorts very often. Additionally, research among Hispanic American women has shown that they are more likely to self-sexualize in Myspace profile pictures than White and Black women (Hall, West, \& McIntyre, 2012), which the researchers aligned with the Hispanic culture as typically hypermasculine and hyperfeminine. Spanish movies have, for example, been argued to portray men in stereotypical ways (e.g., Fouz- 
Herdnández \& Martínez-Expósito, 2007). Spanish men are often depicted as the macho ibérico, the young athletic ideal, or as the muscular hero, characterized by his strong muscular ideal body and objectification of women. Such findings indicate that Spanish adolescents might grow up in a more sexual culture, which could underlie the current findings to some extent. However, more research is necessary to ascertain whether these findings actually indicate cross-cultural differences. More specifically, Spanish adolescents in our study reported to use social media most frequently, which might have reduced variability and could also explain the insignificant findings.

A second conclusion that can be drawn from the cultural perspective is that reading women's magazines was directly related to engagement in self-sexualizing appearance behaviors, among girls in Spain, Belgium, and South-Korea. Although the current study did not include measures on the specific content that girls are exposed to when reading magazines, the fact that girls in those three countries were more inclined to engage in appearance behaviors could tentatively indicate that women's magazines in different countries may carry out the same message, namely that having and maintaining an ideal female appearance (even if the conceptualization of an ideal female appearance may differ between countries; Moradi, 2010; Yan \& Bissell, 2014) is important and normative.

A final conclusion relates to the inclusion of a rewards perspective on the prevailing appearance ideals. Specifically, our findings indicated that (only) prime time television and IRAI were significantly related among Spanish, Belgian, and South-Korean adolescents. In addition, it appeared that IRAI and self-sexualizing behaviors were only significantly related among all the girls in all countries - which provides an answer to our third research question. This finding and the null-finding among boys (with the exception of Belgian boys) coincide with the salience of rewards for girls who engage in self-sexualizing appearance behaviors in society. Girls' tendency to buy into the idea that looking attractive would bring about certain 
benefits, such as happiness or popularity, increased their inclination to behave accordingly.

However, it should be noted that although female attractiveness has been depicted as a factor contributing to positive life outcomes across media, such as Hollywood films (Smith,

McIntosh, \& Bazzini, 1999), magazines (Malkin et al., 1999), and social media (e.g., Ramsey \& Horan, 2017), the current findings did not find a consistent association between girls' media use and IRAI. Possibly, this finding could have been caused by a power issue. Therefore, future research is warranted to be able to accurately ascertain whether girls' consumption of media increases their inclination to internalize the rewards associated with attractiveness and, in turn, their engagement in appearance behaviors.

\section{Implications for Theory and Practice}

The current findings may have important implications for both theory and practice. First, they attest to the value of taking a rewards perspective to understand adolescents' decision to engage in self-sexualizing appearance behaviors. By incorporating body image theory (Thompson \& Stice, 2001) with social cognitive theory on gender development (Bussey \& Bandura, 1999), the current research empirically corroborates the recurring claim that adoption of (gender-linked) behaviors is triggered by reinforcements. However, simultaneously the current study reveals that some individuals (i.e., girls and early adolescents) may be more vulnerable to this process than others (i.e., boys and middle adolescents). We believe this finding might therefore entail a valuable practical implication of the current study. Specifically, we argue that intervention initiatives would benefit from taking into account the internalization of the rewards that may be associated with fitting the ideal mold and to focus specifically on girls and early adolescents.

Second, the current study adds to the literature by exploring the relative contribution of specific media genres to self-sexualization. A recent meta-analysis (Karsay et al., 2017) already indicated that online media lead to stronger self-objectification effects compared to 
television use among men and women. This conditional effect of media type was also present in the current study. Specifically, the consistent association between social media use and self-sexualization across countries demonstrates the unique aspects of social media in which information is mostly produced by peers, rendering it especially relevant and real to adolescents. However, research indicates the idealized imagery on social media (e.g., Manago, Graham, Greenfield, \& Salimkhan, 2008) and thus adolescents, parents, and educators should be made aware of the unrealistic nature of the content and the almost exclusive focus on positive stories/images on social media.

Related to the above, the current study adds to a growing body of work on media sexualization by expanding to the behaviorial aspect of sexualization (Ward et al., 2015). Within the literature on media sexualization, the most prominent area has been focused on whether media exposure affects how people see themselves and their bodies (Ward, 2016). The current study adds that adolescents could become more inclined to engage in various appearance-focused behaviors to emulate a (sexualized) ideal. We ask for further scholarly attention on adolescents' behavioral investment in their appearance, for two reasons. More specific, although adolescents may not intend to look sexually appealing when they wear short skirts/shorts or tight clothes, such behaviors may still be considered inappropriate to their age (Gunter, 2014) and may lead the way to more deliberate self-sexualization when they grow older. Second, in light of self-perception theory (Bern, 1972), adolescents could further infer attitudes and beliefs from observing themselves engaging in such behaviors and thus trigger reciprocity in the examined relations. Although our results found support for inverse relations, future research applying a longitudinal or experimental design is warranted to ascertain the temporal order of the associations between media use, IRAI, and selfsexualizing behaviors. 


\section{Limitations}

The current findings should be interpreted in light of some limitations. First, as mentioned earlier, the current study used a cross-sectional design which precludes us from making any causal inferences. Second, the current study relied on self-report measures. It should be acknowledged that adolescents might have had difficulty estimating their time spent on social and traditional media, considering that media use is regarded as a leisure activity and individuals generally do not actively register how much time they spend on using it (Van den Bulck \& Hofman, 2009). Therefore, self-report data on media use is likely to be sensitive to response biases and underestimation. Lastly, our measures of media exposure were all based on one-item questions pertaining to overall amount of exposure. Such measures were chosen to increase generalizability across countries, which would have been more difficult to establish if media content that is broadcast/read in each country was measured. However, future studies may want to include more diverse media measures to increase understanding of what type of content adolescents are consuming and, perhaps more importantly, how they interact with that content. Moreover, measures are necessary to more explicitly tap into adolescents' exposure to certain media messages. Although sexualizing and beauty-is-good messages have been shown to occur across various media genres (e.g., Kim et al., 2007; Northup \& Liebler, 2010, our measurement precludes us from making inferences about the influence of exposure to these specific messages on adolescents' self-sexualization.

\section{Conclusion}

The present study contributes to a deeper understanding of the association between different types of media use and youth's self-sexualization. Social media use and women's magazines seemed uniquely related to adolescents' behavioral self-sexualization. Only among girls and early adolescents, it was found that the extent to which they believed that an ideal and attractive appearance is rewarded, related to their engagement in self-sexualizing 
behaviors. By revealing that girls and early adolescents ( $<12$ years old) are particularly likely to buy into the idea of rewards for attractiveness and to engage in behaviors to emulate an ideal, the present study contributes to the identification of potential risk groups whom intervention programs should target. The findings further provide evidence for cultural similarities by showing that social media use was directly related to boys' and girls' selfsexualization in all countries, warranting future research examining the influence of sexualizing peer images on adolescents' body image and appearance-related behaviors. 


\section{References}

American Psychological Association. (2007). Report of the APA Task Force on the sexualization of girls. Washington DC: American Psychological Association.

Arnett, J. J. (1995). Adolescents' use of media for self-socialization. Journal of Youth and Adolescence, 24, 519-533. doi: 10.1007/BF01537054

Aubrey, J. S., \& Frisby, C. M. (2011). Sexual objectification in music videos: A content analysis comparing gender and genre. Mass Communication and Society, 14, 475-501. doi: $10.1080 / 15205436.2010 .513468$

Barlett, C. P., Vowels, C. L., \& Saucier, D. A. (2008). Meta-Analyses of the Effects of Media Images on Men's Body-image Concerns. Journal of Social and Clinical Psychology, 27, 279-310. doi: 10.1521/jscp.2008.27.3.279

Bern, D. J. (1972). Self-perception theory. In L. Berkowitz (Ed.), Advances in experimental social psychology (Vol. 6, pp. 1-62). San Diego, CA: Academic Press.

Bielby, D. D., \& Harrington, C. L. (2008). Global TV: Exploring television and culture in the world market. New York University Press: New York.

Blakemore, S-J., \& Mills, K. L. (2014). Is adolescence a sensitive period for sociocultural processing? Annual Review of Psychology, 65, 187-207. doi: 10.1146/annurev-psych010213-115202

Bussey, K., \& Bandura, A. (1999). Social cognitive theory of gender development and differentiation. Psychological Review, 106, 676-713.

Cheung, G. W., \& Rensvold, R. B. (2002). Evaluating Goodness-of-Fit Indexes for testing measurement invariance. Structural Equation Modeling: A Multidisciplinary Journal, 9, 233-255. doi: 10.1207/S15328007SEM0902

Cohen, R., \& Blaszczynski, A. (2015). Comparative effects of Facebook and conventional 
media on body image dissatisfaction. Journal of Eating Disorders, 3, 1-11. doi: $10.1186 / \mathrm{s} 40337-015-0061-3$

Connolly, J., \& McIsaac, C. (2011). Romantic relationships in adolescence. In M. K. Underwood \& L. H. Rosen (Eds.), Social development: Relationships in infancy, childhood,and adolescence (pp. 180-203). New York, NY: Guilford Press.

Cunningham, M., Roberts, A., Barbee, A., Druen, P., \& Wu, C. (1995). Their ideas of beauty are, on the whole, the same as ours: Consistency and variability in the cross-cultural perception of female attractiveness. Journal of Personality and Social Psychology, 68, $261-279$.

Engeln-Maddox, R. (2006). Buying a beauty standard or dreaming of a new life? Expectations associated with media ideals. Psychology of Women Quarterly, 30, 258266. doi: 10.1111/j.1471-6402.2006.00294.x

Erikson, E. H. (1968). Youth, identity, and crisis. New York: Norton.

Fouz-Hernández, S., \& Martínez-Expósito, A. (2007). Live flesh: The male body in contemporary Spanish cinema. I.B. Tauris: London.

Frisby, C. M., \& Aubrey, J. S. Race and genre in the use of sexual objectification in female artists' music videos. Howard Journal of Communications, 23, 66-87. doi: $10.1080 / 10646175.2012 .641880$

Frison, E., \& Eggermont, S. (2015). Exploring the relationships between different types of Facebook use, perceived online social support, and adolescents' depressed mood. Social Science Computer Review, 45, 1-19. doi: 10.1177/0894439314567449

Galambos, N. L., Berenbaum, S. A., \& McHale, S. M. (2009). Gender development in adolescence. In R. M. Lerner \& L. Steinberg (Eds.), Handbook of adolescentpPsychology: Vol 1: Individual bases of adolescent development (3rd ed., pp. 305-357). Hoboken, NJ: John Wiley \& Sons. 
Ganahl, D. J., Prinsen, T. J., \& Netzley, S. B. (2003). A content analysis of prime time commercials: A contextual framework of gender representation. Sex Roles, 49, 545-551. doi: 10.1023/A:1025893025658

Gattino, S., de Piccoli, N., Fedi, A., Boza, M., \& Rollero, C. (2017). A cross-cultural study of biological, psychological, and social antecedents of self-objectification in Italy and Romania. Sex Roles, 1-13. doi: 10.1007/s11199-017-0804-5

Gooderham, P. N., \& Nordhaug, O. (2002). The decline of cultural differencesin Europe. European Banking Federation, 8, 48-53.

Graff, K. A., Murnen, S. K., \& Krause, A. K. (2013). Low-cut shirts and high-heeled shoes: Increased sexualization across time in magazine depictions of girls. Sex Roles, 69, 571582. doi: 10.1007/s11199-013-0321-0

Hall, P. C., West, J. H., \& McIntyre, E. (2012). Female self-sexualization in Myspace.com personal profile photographs. Sexuality and Culture, 16, 1-16. doi: 10.1007/s12119-0119095-0

Harrison, K., \& Fredrickson, B. L. (2003). Women's sports media, self-objectification, and mental health in Black and White adolescent females. Journal of Communication, 53, 216-232. doi: 10.1111/j.1460-2466.2003.tb02587.x

Hatton, E., \& Trautner, M. N. (2011). Equal opportunity objectification? The sexualization of men and women on the cover of Rolling Stone. Sexuality and Culture, 15, 256-278. doi: $10.1007 / \mathrm{s} 12119-011-9093-2$

Hill, J. P., \& Lynch, M. E. (1983). The intensification of gender-related role expectations during early adolescence. In J. Brooks-Gunn \& A. C. Peterson (Eds.), Girls at puberty (pp. 201-228). New York: Plenum Press.

Hofstede, G. (1983). The cultural relativity of organizational practices and theories. Journal of International Business Studies, 75-89. 
Hofstede, G. (2001). Culture's consequences. Comparing values, behaviors, institutions, and organizations across nations. Thousand Oaks, CA: SAGE Publications, Inc.

Holland, G., \& Tiggemann, M. (2016). A systematic review of the impact of the use of social networking sites on body image and disordered eating outcomes. Body Image, 17, 100-110. doi: /10.1016/j.bodyim.2016.02.008

Jones, D. C., Vigfusdottir, T. H., \& Lee, Y. (2004). Body image and the appearance culture among adolescent girls and boys: An examination of friend conversations, peer criticism, appearance magazines, and the internalization of appearance Ideals. Journal of Adolescent Research, 19, 323-339.doi: 10.1177/0743558403258847

Kapidzic, S., \& Herring, S. C. (2014). Race, gender, and self-presentation in teen profile photographs. New Media \& Society, 17, 1-19. doi: 10.1177/1461444813520301

Karsay, K., Knoll, J., \& Matthes, J. (2017). Sexualizing media use and self-objectification: A meta-analysis. Psychology of Women Quarterly. Advanced online publication. doi: $10.1177 / 0361684317743019$

Kim, J. L., Sorsoli, C. L., Collins, K., Zylbergold, B. a, Schooler, D., \& Tolman, D. L. (2007). From sex to sexuality: Exposing the heterosexual script on primetime network television. Journal of Sex Research, 44, 145-57. doi: 10.1080/00224490701263660

Kim, K. B., \& Aubrey, J. S. (2015). A cross-cultural comparison of cognitive and affective mediators in the relationship between media use and body image disturbance: Focusing on US and Korean women. Asian Journal of Communication, 25, 507-524. doi: $10.1080 / 01292986.2014 .995681$

Kroger, J. (2007). Identity development. Adolescence through adulthood. Thousand Oaks: Sage.

Lee, H-R., Lee, H. E., Choi, J., Kim, J. H., \& Han, H. L. (2014). Social media use, body image, and psychological well-being: A cross-cultural comparison of Korea and the United States. Journal of Health Communication, 19, 1343-1358. doi: 
$10.1080 / 10810730.2014 .904022$

Lee, G., Lee, J., \& Kwon, S. (2011). Use of socialnetworking sites and subjective wellbeing: A study in South Korea. Cyberpsychology, Behavior, and Social Networking, 14, 151155. doi: 10.1089/cyber.2009.0382

Levine, M. P., \& Murnen, S. K. (2009). “Everybody Knows That Mass Media Are/Are Not [pick one] a Cause of Eating Disorders": A Critical Review of Evidence for a Causal Link Between Media, Negative Body Image, and Disordered Eating in Females. Journal of Social and Clinical Psychology, 28, 9-42. doi: 10.1521/jscp.2009.28.1.9

Madden, M. (2013). Teens haven't abandoned Facebook (yet). Washington, DC: Pew Research Center's Internet \& American Life Project.

Malkin, A. R., Wornian, K., \& Chrisler, J. C. (1999). Women and weight: Gendered messages on magazine covers. Sex Roles, 40, 647-655. doi: 10.1023/A:1018848332464

Manago, A. M., Graham, M. B., Greenfield, P. M., \& Salimkhan, G. (2008). Selfpresentation and gender on MySpace. Journal of Applied Developmental Psychology, 29, 446-458. doi: 10.1016/j.appdev.2008.07.001

Manago, A. M., Ward, L.M. , Lemm, K. M., Reed, L., \& Seabrook, R. (2015). Facebook involvement, objectified body consciousness, body shame, and sexual assertiveness in college women and men. Sex Roles, 72, 1-14. doi: 10.1007/s11199-014-0441-1

Martin, K. A., \& Kayzak, E. (2009). Hetero-romantic love and heterosexiness in children's G-rated films. Gender \& Society, 23, 315-336. doi: 10.1177/0891243209335635

McCabe, M., \& Ricciardelli, L. (2006). A prospective study of extreme weight change behaviors among adolescent boys and girls. Journal of Youth and Adolescence, 35, 425434. doi: 10.1007/s10964-006-9062-5

McKenney, S. J., \& Bigler, R. S. (2014a). Internalized sexualization and its relation to 
sexualized appearance, body surveillance, and body shame among early adolescent girls. The Journal of Early Adolescence, 1-27. doi: 10.1177/0272431614556889

McKenney, S. J., \& Bigler, R. S. (2014b). High heels, low grades: Internalized sexualization and academic orientation among adolescent girls. Journal of Research on Adolescence, 26, 30-36.doi: 10.1111/jora.12179

Moradi, B. (2010). Addressing gender and cultural diversity in Body image : Objectification theory as a framework for integrating theories and grounding research. Sex Roles, 63, 138-148. doi: 10.1007/s11199-010-9824-0

Morris, P. K., \& Nichols, K. (2013). Conceptualizing beauty: A content analysis of US and French women's fashion magazine advertisements. Online Journal of Communication and Media Technologies, 3, 49-74

Neumark-Sztainer, D., \& Hannan, P. J. (2000). Weight-related behaviors among adolescent girls and boys. Results from a national survey. Archives of Pediatrics and Adolescent Medicine, 154, 569-577. doi:10.1001/archpedi.154.6.569

Paek, H. J., Nelson, M. R., \& Vilela, A. M. (2011). Examination of gender-role portrayals in television advertising across seven countries. Sex Roles, 64, 192-207. doi: 10.1007/s11199-010-9850-y

Parent, A-S., Teilmann, G., Juul, A., Shakkebaek, N. E., Toppari, J., \& Bourguignon, J-P. (2003). The timing of normal puberty and the age limits of sexual precocity: Variations around the world, secular trends, and changes after migration. Endocrine Reviews, 24, 668-693. doi: 10.1210/er.2002-0019

Park, N., Song, H., \& Lee, K. M. (2014). Social networking sites and other media use, acculturation stress, and psychological well-being among East Asian students in the United States. Computers in Human Behavior, 36, 138-146. doi:

10.1016/j.chb.2014.03.037 
Perloff, R. M. (2014). Social media effects on young women's body image concerns:

Theoretical perspectives and an agenda for research. Sex Roles, 71, 363-377. doi: 10.1007/s11199-014-0384-6

Pew Research Center (2015). Social media usage: 2005-2015. Retrieved from assets.pewresearch.org/wp-content/uploads/sites/14/2015/10/PI_2015-10-08_SocialNetworking-Usage-2005-2015_FINAL.pdf

Priess, H. A., Lindberg, S. M., \& Hyde, J. S. (2009). adolescent gender-role identity and mental health: Gender intensification revisited. Child Development, 80, 1531-1544. doi: 10.1111/j.1467-8624.2009.01349.x

Ramsey, L. R., \& Horan, A. L. (2017). Picture this: Women's self-sexualization in photos on social media. Personality and Individual Differences. doi: 10.1016/j.paid.2017.06.022

Ricciardelli, R., Clow, K. A., \& White, P. (2010). Investigating hegemonic masculinity: Portrayals of masculinity in men's lifestyle magazines. Sex Roles, 63, 64-78. doi: $10.1007 / \mathrm{s} 11199-010-9764-8$

Smith, S., McIntosh, W., \& Bazzini, D. (1999). Are the beautiful good in Hollywood? An investigation of the beauty-and-goodness stereotype on film. Basic and Applied Social Psychology, 21, 69-80. doi: 10.1207/s15324834basp2101_7

Smolak, L., Murnen, S. K., \& Myers, T. A. (2014). Sexualizing the Self: What College Women and Men Think About and Do to Be "Sexy." Psychology of Women Quarterly, 38, 379-397. doi: 10.1177/0361684314524168

Speno, A. G., \& Aubrey, J. S. (2017). Sexualization, youthification, and adultification : A content analysis of images of girls and women in popular magazines. Journalism and Mass Communication Quarterly, 1-22. doi: 10.1177/1077699017728918 
Stice, E. (2002). Risk and maintenance factors for eating pathology: A meta-analytic review. Psychological Bulletin, 128, 825-848. doi: 10.1037/0033-2909.128.5.825

Strasburger, V. C., \& Wilson, B. J. (2002). Youth and media: Opportunities for development or lurking dangers? Children, Adolescents, and the Media. Thousand Oaks, CA: Sage.

Subrahmanyam, K., \& Greenfield, P. (2016). Online communication and adolescent relationships. The Future of Children, 18, 119-146. doi: 10.1353/foc.0.0006

Sypeck, M. F., Gray, J. J., \& Ahrens, A. H. (2004). No longer just a pretty face: Fashion magazines ' depictions of ideal female beauty from 1959 to 1999. International Journal of Eating Disorders, 36, 342-347. doi: 10.1002/eat.20039

Thompson, J. K., \& Stice, E. (2001). Thin-Ideal Internalization: Mounting Evidence for a New Risk Factor for Body-Image Disturbance and Eating Pathology. Current Directions in Psychological Science, 10, 181-183. doi: 10.1111/1467-8721.00144

Thompson, J. K., Van Den Berg, P., Roehrig, M., Guarda, A. S., \& Heinberg, L. J. (2004). The Sociocultural Attitudes Towards Appearance Scale-3 (SATAQ-3): Development and validation. International Journal of Eating Disorders, 35, 293-304. doi: 10.1002/eat. 10257

Tod, D., \& Edwards, C. (2013). Predicting drive for muscularity behavioural engagement from body image attitudes and emotions. Body Image, 10, 135-138. Doi: 10.1016/j.bodyim.2012.08.010

Trekels, J., Eggermont, S. (2016). Beauty is Good: The appearance culture, the internalization of appearance ideals, and dysfunctional appearance beliefs among tweens. Human Communication Research, 43, 173-192. doi: 10.1111/hcre.12100

Trekels, J., Eggermont, S. (2017a). Linking Magazine Exposure to Social Appearance 
Anxiety: The Role of Appearance Norms in Early Adolescence. Journal of Research on Adolescence. Online first publication. doi: 10.1111/jora.12311

Trekels, J., Eggermont, S. (2017b). Aspiring to have the looks of a celebrity: Young girls' engagement in appearance management behaviors. European Journal of Pediatrics, 176, 857-863. doi: 10.1007/s00431-017-2918-8

Trekels, J., \& Eggermont, S. (2017c). "I can/should look like a media figure.” The association between direct and indirect media exposure and teens' sexualizing appearance behaviors. Journal of Sex Research. doi: 10.1080/00224499.2017.1387754

Tsitsika, A. K., Tzavela, E. C., Janikian, M., Ólafsson, K., Iordache, A., Schoenmakers, T. M., Tzavara, C., \& Richardson, C. (2014). Online social networking in adolescence: Patterns of use in six European Countries and links with psychosocial functioning. Journal of Adolescent Health, 55, 141- 147. doi: 10.1016/j.jadohealth.2013.11.010

Utz, S., Muscanell, N., \& Cameran, K. (2015). Snapchat elicits more jealousy than Facebook: A comparison of Snapchat and Facebook use. Cyberpsychology, Behavior, and Social Networking, 18, 141-146. doi: 10.1089/cyber.2014.0479

Vandenbosch, L., \& Eggermont, S. (2012). Understanding sexual objectification: A comprehensive approach toward media exposure and girls' internalization of beauty ideals, self-objectification, and body surveillance. Journal of Communication, 62, 869887. doi: 10.1111/j.1460-2466.2012.01667.x

Vandenbosch, L., \& Eggermont, S. (2015). Sexualization of adolescent boys: Media exposure and boys' internalization of appearance ideals, self-objectification, and body surveillance. Men and Masculinities, 16, 283-306. doi: 10.1177/1097184X13477866

Van den Bulck, J., \& Hofman, A. (2009). The Television-to-Exercise Ratio is a Predictor of Overweight in Adolescents: Results from a Prospective Cohort Study with a Two Year Follow Up. Preventive Medicine, 48, 368-371. 
Vereecken, C. A., Todd, J., Roberts, C., Mulvihill, C., \& Maes, L. (2006). television viewing beahviour and associations with food habits in different countries. Public Health Nutrition, 9, 244-250. doi: 10.1079/PHN2005847

Ward, L. M. (2016). Media and sexualization: State of empirical research, 1995-2015. Journal of Sex Research, 53, 560-577. doi: 10.1080/00224499.2016.1142496

Ward, L. M., \& Harrison, K. (2005). The impact of media use on girls' beliefs about gender roles, their bodies, and sexual relationships: A research synthesis. In E. Cole \& J. H. Daniels (Eds.), Featuring females: Feminist analyses of media (pp. 3-23). Washington, DC: American Psychological Association.

Ward, L. M., Seabrook, R. C., Manago, A., \& Reed, L. (2015). Contributions of diverse media to self-sexualization among undergraduate women and men. Sex Roles, 12-23. doi: 10.1007/s11199-015-0548-Z

Williams, J. M., \& Currie, C. (2000). Self-esteem and physical development in early adolescence: Pubertal timing and body image. Journal of Early Adolescence, 20, 129149. doi: $10.1177 / 0272431600020002002$

Yan, Y., \& Bissell, K. (2014). The globalization of beauty: How is ideal beauty influenced by globally published fashion and beauty magazines? Journal of Intercultural Communication Research, 43, 194-214. doi: 10.1080/17475759.2014.917432

Zsakai, A., Karkus, Z., Ultczas, K., \& Bodzsar, E. V., (2017). Body structure and physical self-concept in early adolescence. Journal of Early Adolescence, 37, 316-338. doi: $10.1177 / 0272431615602757$

Zurbriggen, E., \& Roberts, T. (2013). The sexualization of girls and girlhood: Causes, consequences, and resistance. Oxford University Press: New York. 
Table 1

Descriptive statistics and zero-order correlations

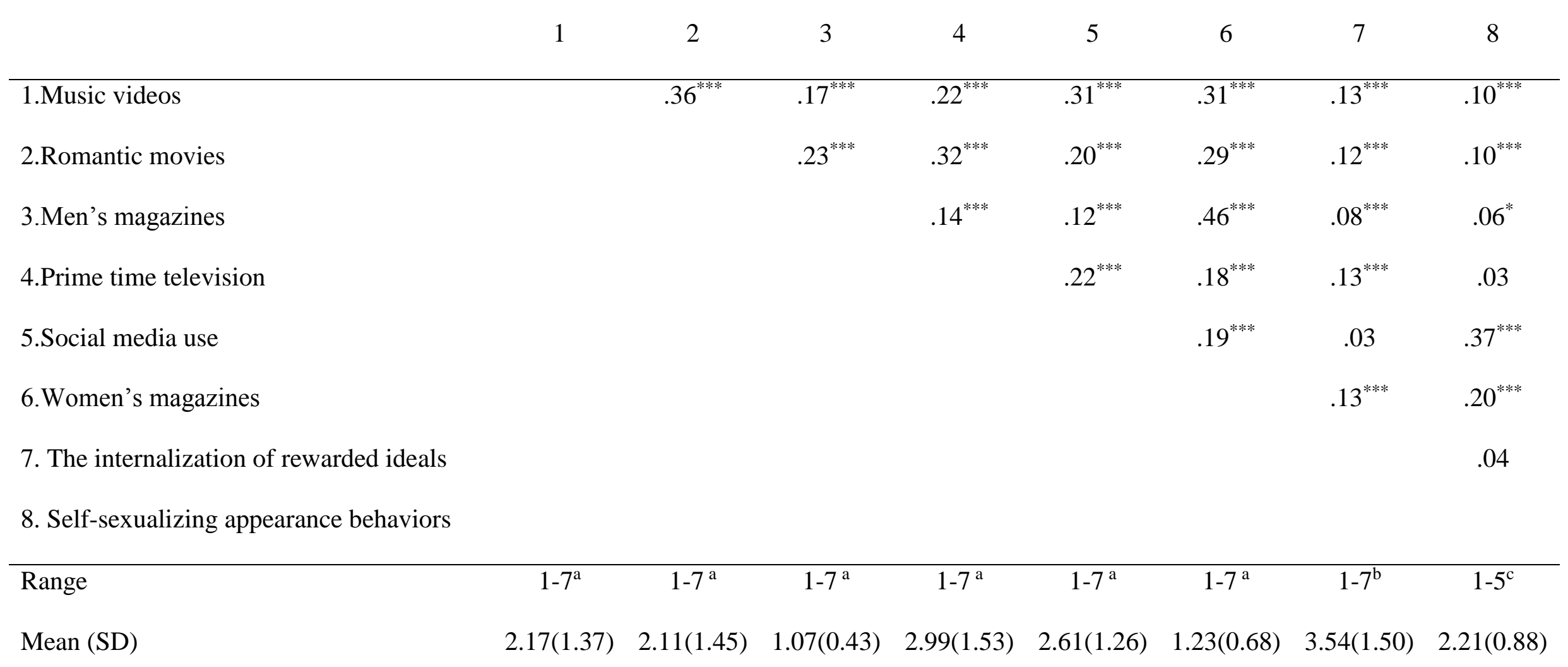

Note. ${ }^{\text {a }}$ = Likert scale ranging from (1) = "never use it to less than 10 min", (2) = "10-30 minutes"; (3) = "31-60 minutes"; (4) = "1-2 hours"; (5) = "3-4 hours"; $(6)=$ "5-6 hours"; (7) = "more than 6 hours"; ${ }^{\text {b }}=$ likert scale ranging from $(1)=$ "I totally disagree" to (5) = "I totally agree"; c = Likert scale ranging from (1) = "never"; (2) = "seldom"; (3) = "sometimes"; (4) = "often"; (5) = "always."

$* p<.05, * * p<.01, * * * \mathrm{p}<.001$ 


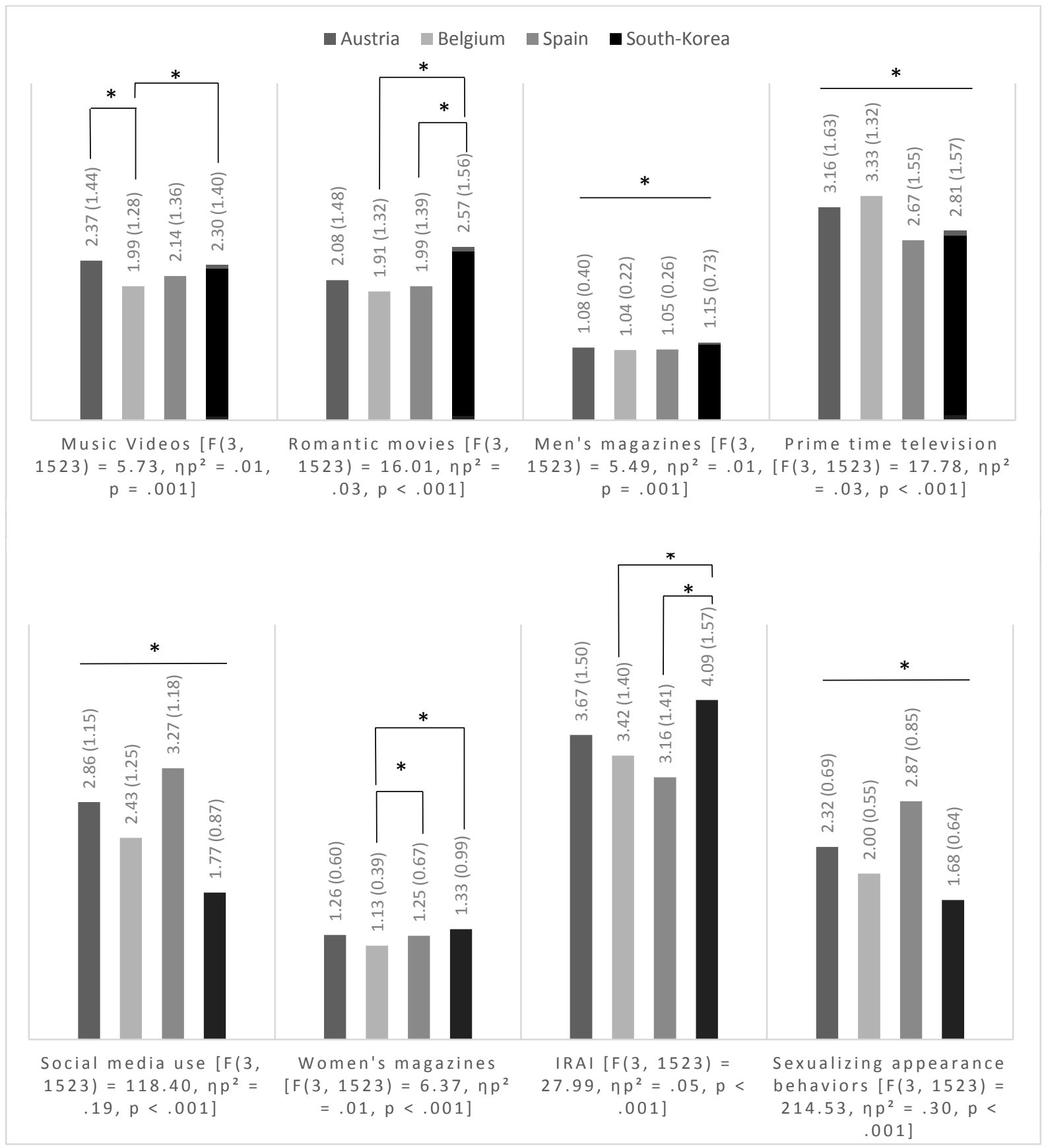

Figure 1. Bar graph model summarizing the MANOVA analysis results for differences between participants from different countries on key variables $(\mathrm{N}=1,527)$.

Note. There were significant differences for all participants with regard to men's magazines, prime time television, social media use, and sexualizing appearance behaviors. 


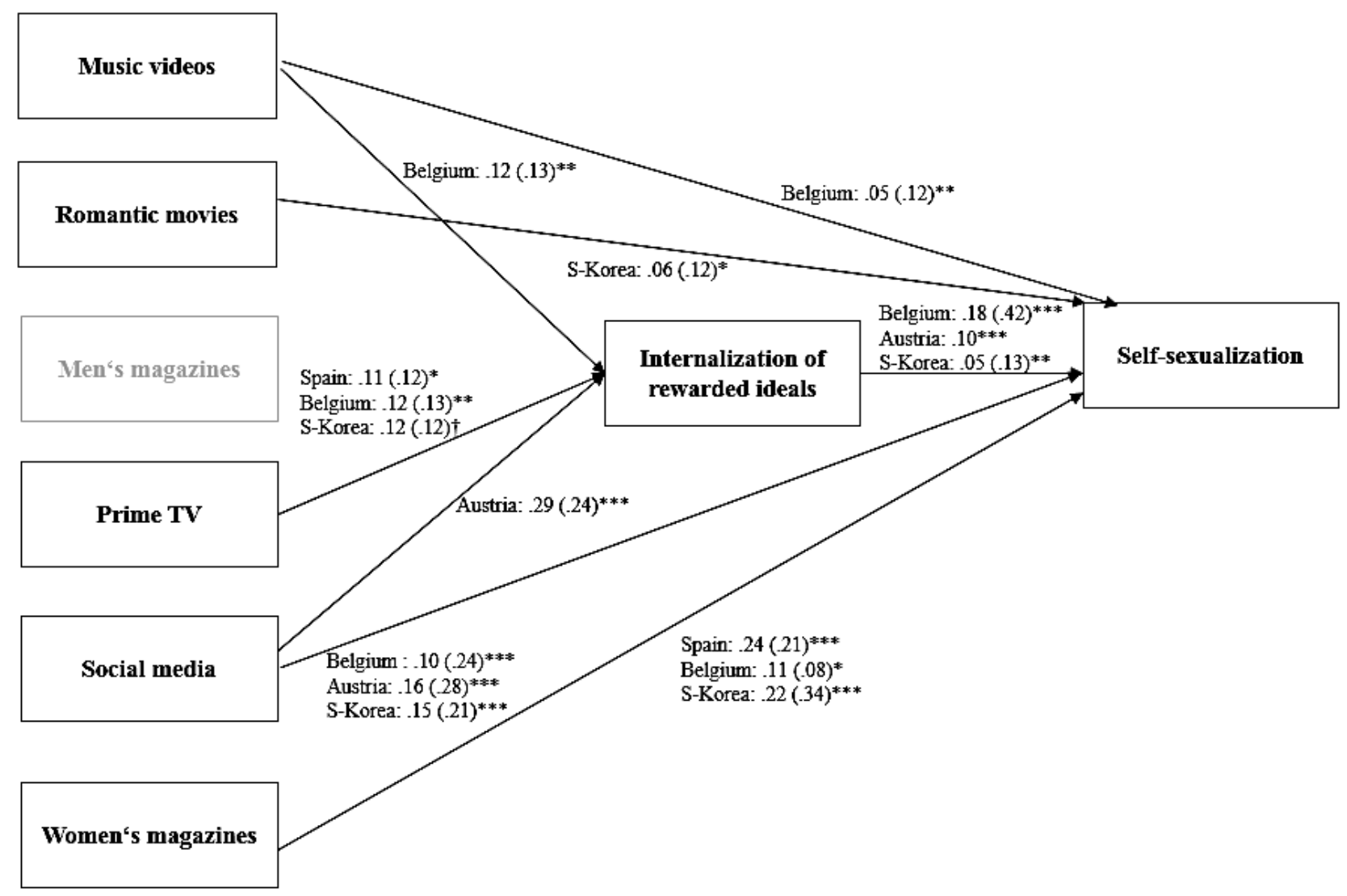

Figure 2. Structural equation model for the relationships between media exposure, the internalization of rewarded appearance ideals, and engagement in self-sexualizing appearance behaviors $(\mathrm{N}=1,527)$.

Note. Values reflect standardized coefficients (beta), values within brackets reflect unstandardized coefficients (b-value).

Only significant paths are shown $(* p<.05, * * p<.01, * * * p<.001)$. 


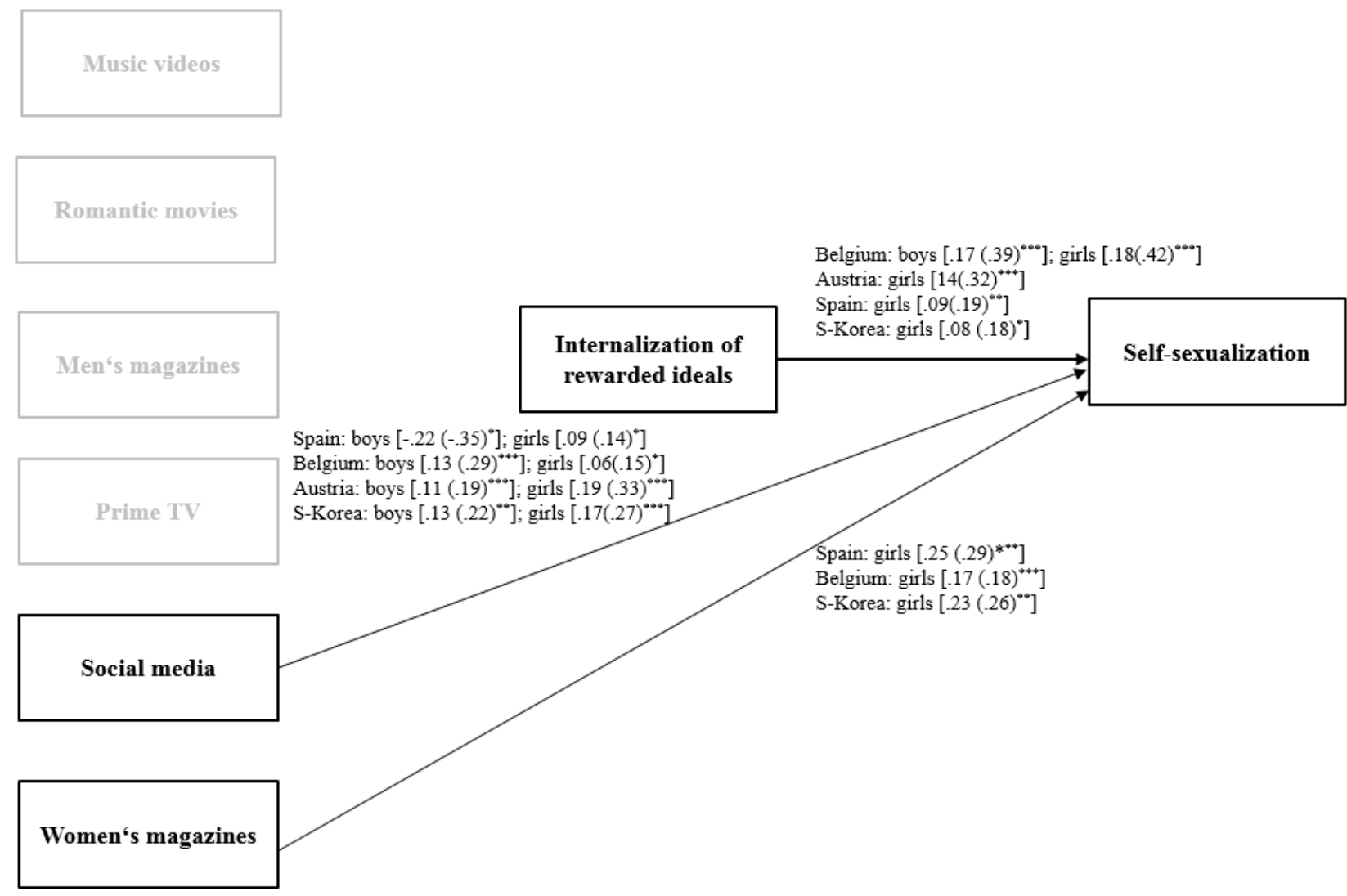

Figure 3. Cross-cultural differences in the relations between media exposure, the internalization of rewarded appearance ideals, and engagement in self-sexualizing appearance behaviors (multiple group analysis).

Note. Values reflect standardized coefficients (beta), values within brackets reflect unstandardized coefficients (b-value).

Only significant different paths are shown $(* p<.05, * * p<.01, * * * p<.001)$. 


\section{Acknowledgements}

\section{Authors' Contributions}

JT conceived of the study, participated in its design and coordination, collected data, performed the statistical analyses, interpreted the results, and drafted the manuscript; KK conceived of the study, participated in its design and coordination, collected data, and critically revised the manuscript; SE conceived of the study, participated in the design and coordination of the study, interpreted the results, and critically revised the manuscript. LV conceived of the study, participated in its design and coordination, collected data, and critically revised the manuscript. All authors read and approved the final manuscript.

\section{Data Sharing Declaration}

The datasets generated and/or analyzed during the current study are not publicly available but are available from the corresponding author on reasonable request.

\section{Conflicts of Interest}

The authors report no conflict of interest.

\section{Compliance with Ethical Standards}

\section{Funding}

The research groups in the participating countries funded the research project with their own research funds.

\section{Ethical Approval}

The ethical committees of each host university approved the research project. 
MEDIA USE, INTERNALIZATION, AND SELF-SEXUALIZING APPEARANCE BEHAVIORS

\section{Informed Consent}

Active consent was obtained from both the parents and adolescents before the respondents filled out the paper-and-pencil surveys. 


\section{Author Affiliations and Research Interests}

Jolien Trekels is a PhD student at the School for Mass Communication Research at the KU Leuven. Her interests include the influence of media exposure on body image among adolescents.

Kathrin Karsay is a postdoctoral researcher at the Department of Communication at the University of Vienna. Her research interests focus on the effects of media use on well being - mainly in relation to body image.

Steven Eggermont is a Professor at the Faculty of Social Sciences at the KU Leuven. His interests include media use during the life course and effects of exposure to the media on perceptions and behaviors.

Laura Vandenbosch is an Assistant Professor at the Faculty of Social Sciences at the KU Leuven. Her research studies the relation between media and adolescent well-being from different perspectives, such as gender socialization, developmental psychology, and body image. 